Article

\title{
Effect of Leptosphaeria maculans Infection on Promoter DNA Methylation of Defence Genes in Brassica napus
}

\author{
Soodeh Tirnaz, Clementine Merce, Philipp E. Bayer@, Anita A. Severn-Ellis® $₫$, David Edwards® \\ and Jacqueline Batley *(B) \\ School of Biological Sciences, University of Western Australia, Perth, WA 6009, Australia; \\ soodeh.tirnaz@research.uwa.edu.au (S.T.); clementine.merce@research.uwa.edu.au (C.M.); \\ philipp.bayer@uwa.edu.au (P.E.B.); anita.severn-ellis@uwa.edu.au (A.A.S.-E); dave.edwards@uwa.edu.au (D.E.) \\ * Correspondence: jacqueline.batley@uwa.edu.au
}

Received: 23 June 2020; Accepted: 22 July 2020; Published: 24 July 2020

\begin{abstract}
Canola (Brassica napus) is an important crop species grown worldwide for its seeds, which are crushed for edible oil. Canola production is threatened by blackleg disease, caused by the fungal pathogen Leptosphaeria maculans, which can lead up to 100\% yield loss. A plant's disease resistance response depends highly on the regulation of the expression of defence genes. DNA methylation, an epigenetic mark, is one of the most important regulatory mechanisms in a plant's defence system. Here, the DNA methylation pattern of promoters of defence genes has been investigated between leaves from control and infected plants with L. maculans of resistant and susceptible cultivars of B. napus. In total, over 12,000 promoters were found to be differentially methylated between leaves from control and infected plants in the two cultivars, of which 225 promoters were related to defence genes and differentially methylated between the resistant and susceptible cultivars. The majority of defence gene promoters were hypo- or hyper-methylated in the first and second true leaves, but not in the third and fourth leaves. The outcomes will assist in developing an insight into genome-wide DNA methylation patterns in canola cultivars, and ultimately help breeders to optimise the breeding programmes for enhancing resistance against blackleg disease.
\end{abstract}

Keywords: Brassicaceae; Brassica napus; blackleg; epigenetics; resistance gene

\section{Introduction}

Brassica napus (canola, rapeseed, and oilseed rape) is cultivated as the most productive Brassica oilseed species, and is grown primarily for its seed. B. napus originated from interspecific hybridisation between the diploids B. rapa (A genome) and B. oleracea (C genome), resulting in an amphidiploid genome (AACC, $n=19$ ) [1]. Worldwide canola production is threatened by various diseases, including stem rot, white rust, clubroot, downy mildew and blackleg. Blackleg, caused by the fungal pathogen Leptosphaeria maculans, is the most devastating disease of oilseed Brassica species, particularly B. napus in Australia, Canada and Europe [2], which can cause up to $100 \%$ yield losses [3].

Early stages of infection occur at the seedling stage and symptoms appear as cotyledon and leaf necrotic lesions; then, from the infected leaves, the fungus grows within the vascular tissue to the stem base, causing a basal canker at the adult growth stage [4]. Blackleg damage can be partially controlled using strategies such as fungicide applications and crop rotation; however, growing resistant cultivars of B. napus is considered the most promising disease management strategy [2]. Resistance responses in B. napus against blackleg occur both at the cotyledon and adult stages with the involvement of large numbers of resistance genes. Nucleotide-binding site leucine-rich repeat (NLR), receptor-like kinases 
(RLKs), and membrane associated receptor-like proteins (RLPs) are the main classes of resistance genes and are known as resistance gene analogs (RGAs) [5].

B. napus resistance to blackleg at the cotyledon stage is a typical example of a plant pathogen-derived effector-triggered immunity (ETI) immunity system [6]. The ETI system is a gene for gene resistance in which a resistance (R) gene in the host plant has a corresponding avirulence (Avr) gene in the fungus $[7,8]$. While NLRs are known as R genes and initiate ETI, RLKs and RLPs initiate the first layer of plant innate immunity, known as PAMP-triggered immunity (PTI) [9]. In addition to the presence of a resistance gene, the resistance response depends on a plant's ability to reprogram gene expression in response to pathogen attack; therefore, the presence of resistance genes is not a guarantee of a resistant response.

There are several mechanisms involved in transcriptional reprogramming, including epigenetic modifications, such as DNA methylation and chromatin modification and remodeling [10,11]. DNA methylation is an important and common epigenetic modification of DNA in eukaryotes [12] and is involved in diverse biological processes, including genome evolution and developmental regulation [12]. Defending the genome against transposons and regulating gene expression have been named as two essential roles of DNA methylation [13]. DNA methylation refers to the addition of a methyl group to the cytosine bases of DNA to form 5-methylcytosine [14], and in plants, methylation of cytosine bases is observed in the context of CG (cytosine nucleotide followed by a guanine), CHG (cytosine, followed by adenine, cytosine, or thymine, followed by a guanine) and CHH (cytosine, followed by two non-guanine nucleotides) [15].

DNA methylation through gene expression regulation impacts on plant adaptation mechanisms in response to both biotic and abiotic stresses [16-18]. The correlation between DNA methylation and gene expression regulation depends on various factors, including tissue type and genomic regions, for example, gene body and promoters [19]. Gene body methylation in the CG context has been shown to have a positive correlation with gene expression in common bean, soybean [20] and rice [21,22]. A number of studies have confirmed the involvement of pathogen-induced DNA methylation changes in promoters of defence genes, such as NLRs, in the resistance response. For example, in rice hypo- and hyper- methylation in the promoters of NLRs occurs under pathogen attack, such as from Magnaporthe grisea $[23,24]$ and Xanthomonas oryzae pv. orayza [25], which results in transcriptome reprograming and a resistance response. There are also a number of studies confirming the involvement of DNA methylation modification in tolerance improvement under abiotic stresses such as under salinity stress in rice [26] and under heat stress in B. rapa [27]. Due to constant exposure to stress over several generations, an epigenetic modification could convert into a stable (epi) genetic trait of tolerance or resistance [28].

In this study, we investigate the DNA methylation modification of promoters of resistance related genes, particularly RGAs, in B. napus susceptible and resistant cultivars in response to blackleg disease. We reveal the potential role of DNA methylation in $B$. napus resistance responses, which may be employed by breeders for improving disease resistance.

\section{Materials and Methods}

\subsection{Plant Materials and L. maculans Isolate}

Two B. napus spring cultivars were selected, one susceptible (Westar) and one resistant (Sturt) to L. maculans. The resistance phenotype of Sturt was previously confirmed against isolates of L. maculans carrying AvrLM3 genes; thus, a previously characterised isolate of L. maculans containing the AvrLM3 gene, named as 47A2 (A3), was selected for this experiment. The 47A2 (A3) isolate is known to carry two avirulence genes (AvrLM1, AvrLM3). The isolate was provided by Dr Angela Van De Wouw, School of BioSciences, University of Melbourne, Victoria, Australia. The isolate was subcultured and grown on V8 agar media until production of abundant conidia was observed. Conidia concentration of $1 \times 10^{7} \mathrm{ml}^{-1}$ was used for inoculations. 


\subsection{Sowing and Inoculation}

Plants were sown in multicell $(6 \times 8$ cells $)$ trays placed in a plastic container. The trays were filled with potting mix containing $2.5 \mathrm{~m}^{3}$ fine composted pine bark, $1 \mathrm{~m}^{3}$ coco peat, $5 \mathrm{~m}^{3}$ brown river sand, $10 \mathrm{~kg}$ slow release fertilizer Osmofrom ${ }^{\circledR} \mathrm{NXT} 22 \mathrm{~N}+2.2 \mathrm{P}_{2 \mathrm{O} 5}+9.1 \mathrm{~K}_{2} \mathrm{O}+1.2 \mathrm{MG}+$ trace elements (Everris International B.V., Geldermalsen, Netherlands), $10 \mathrm{~kg}$ Dolomite (CalMag) (Green Life Soil Co., Perth, Australia), $5 \mathrm{~kg}$ gypsum clay breaker, $5 \mathrm{~kg}$ extra fine limestone, $4 \mathrm{~kg}$ iron hepta sulphate and $1 \mathrm{~kg}$ iron chelate. Potting mix was pressured $63^{\circ} \mathrm{C}$ for $30 \mathrm{~min}$. The sown seeds were grown in a phytotron under $18 / 13 \pm 2{ }^{\circ} \mathrm{C}$ day/night and natural light, with regular irrigation.

The inoculation was conducted 14 days after sowing as described by Purwantara et al. (1998) [29]. Briefly, the cotyledons were wounded using sterile forceps and a $10 \mu l$ droplet of spore suspension placed on each wound site. One droplet of water was used as mock inoculation for control samples. To ensure successful infection, the plants were covered for $48-72 \mathrm{~h}$ to increase the humidity level. The temperature was increased to $22^{\circ} \mathrm{C}$. After 3 days, the covers were removed and plants were kept in a controlled environment room (CER) under $22 / 20 \pm 2{ }^{\circ} \mathrm{C}$ day/night, light intensity of $200 \mu \mathrm{MOL}$ and day length of $12 \mathrm{~h}$, with regular irrigation.

\subsection{Sampling}

To find the best time for sampling, both cultivars were inoculated with the pathogen and the infection symptoms were monitored daily. Based on these observations, after 9 days of inoculation the infection symptoms (necrotic lesions) were clearly recognizable in both susceptible and resistant cultivars. After $9 \mathrm{dpi}$, all true leaves of both cultivars, including the 1st to 3rd true leaves of Westar and 1st to 4th true leaves of Sturt, were harvested, snap frozen in liquid Nitrogen and stored at $-80^{\circ} \mathrm{C}$ until further use.

\subsection{DNA Extraction, Library Construction and Sequencing}

Genomic DNA of all 3 and 4 true leaves of the cultivars Westar and Sturt, respectively, were extracted and purified using the DNeasy ${ }^{\circledR}$ Plant Mini Kit (Hilden, Germany). Total DNA was quantified using a Qubit 3.0 Fluorometer with the Qubit dsDNA HS Assay Kit (Invitrogen, Carlsbad, CA, USA). Genomic DNA (1000 ng) was used for sequence-captured library construction based on the SeqCap Epi Enrichment Kit (Roche NimbleGen, Madison, WI, USA). The capture probes were designed based on the company standard; $1 \mathrm{kbp}$ upstream of all genes in the reference genome were selected, then repeat masking was performed using RepeatMasker based on all species in Repbase database [30]. After removing all repeat regions from the upstream regions, the sequences longer than $300 \mathrm{bp}$ were selected and sent to Roche for optimisation and probe construction. For library construction, DNA was sheared to $400-450 \mathrm{bp}$ by sonication. The fragmented DNA was subject to end repair and A-tailing, adaptor ligation, post-ligation clean-up and Dual-SPRI size selection using reagents provided (Roche Nimblegen, Madison, WI, USA). Then, bisulfite conversion of the constructed libraries was performed using the EZ DNA Methylation-Lightning Kit (Zymo Research, Irvine, CA, USA). The bisulfite converted libraries were subjected to precaptured LM-PCR, followed by hybridization with designed probes and washing of uncaptured libraries. Postcaptured LM-PCR was conducted followed by PCR clean-up of libraries. The constructed libraries were quantified with Qubit, and the quality was examined by LabChip GX Touch 24 (PerkinElmer, Waltham, MA, USA). Prepared libraries were sequenced at The Garvan Institute of Medical Research (Darlinghurst, Australia) on an Illumina HiSeqXTEN (paired-end reads, 151 bp).

\subsection{Sequence Analysis}

The raw data were trimmed using Trimmomatic ( $\mathrm{v}$ 0.36) in order to remove adapters and low-quality sequences. The reads were then aligned to the reference genome (concatenation of DARMOR-bzh v1.3 and pangenome contigs) [31,32] using Bismark (v 0.19.0) [33]. The mean coverage 
over all samples was 11X. Differentially methylated sites (bases) were detected with the R package MethylKit ( $\mathrm{v}$ 1.12.0) on the gene promoters (defined as $1 \mathrm{~kb}$ upstream of the transcription start site). In this study, differentially methylated promoters were defined as promoters containing at least one differentially methylated base. Bases were considered differentially methylated if their percent methylation difference was larger than $25 \%$ with a q-value threshold of 0.01 . If the methylation difference value was negative, bases were considered hypo-methylated; otherwise, they were considered hyper-methylated. The methylation pattern of RGA promoters was also specifically analysed in both cultivars. RGA identification was performed based on their domain structure using the RGAugury pipeline [34], and the RGAs were classified as RLKs, RLPs and NLRs. NLRs were also classified to sub classes of TNL (TIR-Nucleotide Binding Site (NBS)-Leucine Rich Repeat (LRR)), CNL (Coiled-Coil (CC)-NBS-LRR), TN (TIR-NBS), TX (TIR with unclassified domains), NL (NBS-LRR), CN (CC-NBS), $\mathrm{N}$ (NBS), TM-CC (transmembrane-CC) and others (genes with disordered domains). The complete list of candidate RGAs is available at doi:10.26182/5dbf848ca28c3 [35].

\section{Results and Discussion}

\subsection{Disease Assessment}

In this study, the sampling was a crucial step as we needed to select a time point at which disease symptoms were clearly distinguishable between resistant and susceptible cultivars before cotyledons died due to aging. The infection symptoms were monitored daily after the inoculation and after 9 days of inoculation (dpi) despite the cotyledons of both cultivars turning yellow as a result of aging; the infection symptoms (necrotic lesions) were clearly recognizable in the susceptible cultivar (Westar) but not in the resistant cultivar (Sturt) (Figure 1). If the experiment proceeded for more than $9 \mathrm{dpi}$, all cotyledons in both cultivars were dead and the infection symptoms were not recognisable.

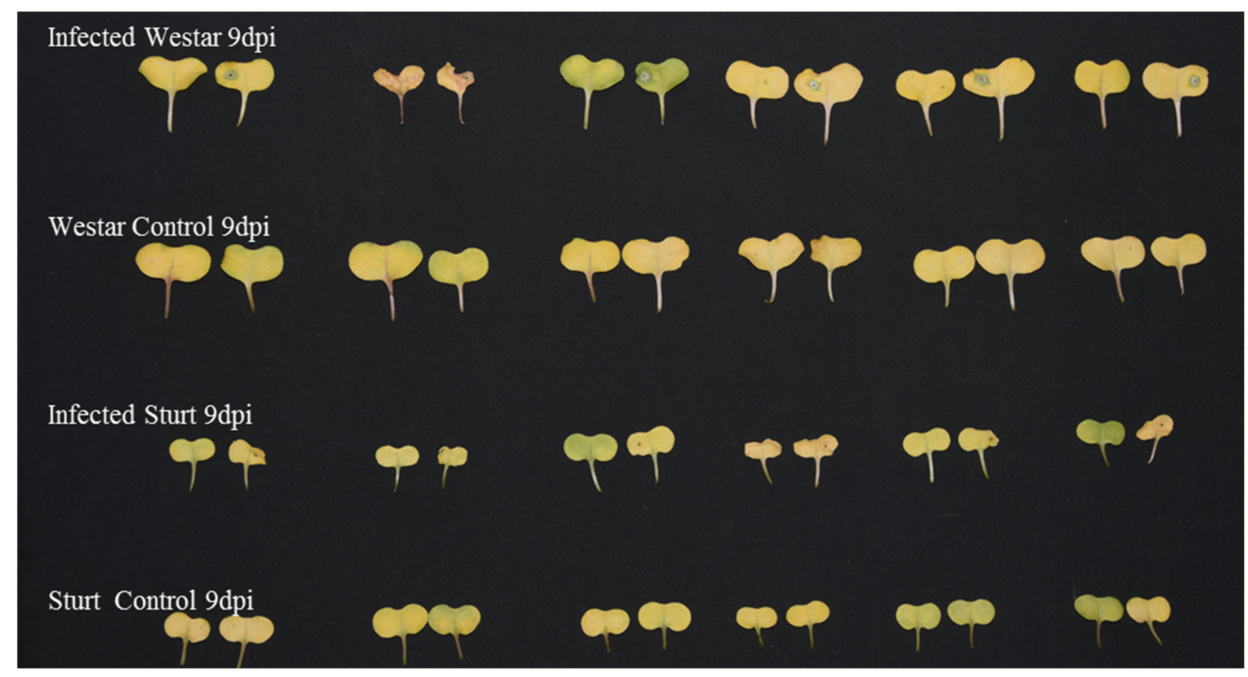

Figure 1. Necrotic lesions caused by Leptosphaeria maculans 9 days after inoculation at the seedling stage of Brassica napus. Susceptible cultivar: Westar, Resistant cultivar: Sturt.

\subsection{Differentially Methylated Promoters in the Cultivars Westar and Sturt Under Pathogen Attack}

A total of 62,583 and 58,100 promoters had enough coverage in Sturt and Westar, respectively, and used for downstream analysis. The comparative analysis between control samples and samples infected with pathogens in both cultivars shows a total of 12,314 promoters were differentially methylated between control and infected samples in both cultivars (Table S1). Out of these 12,314 promoters, 5017 (40.74\%) were differentially methylated between Sturt and Westar; $1775(14.41 \%)$ and 2913 (23.65\%) were differentially methylated only in Westar and Sturt, respectively; and 2609 $(21.2 \%)$ promoters had mixed methylation status (i.e., in some of the leaves they were hypo-methylated 
and in some leaves hyper-methylated) (Figure 2). These observations suggest that in the resistant cultivar Sturt, the occurrence of DNA methylation modification is more dynamic under pathogen attack as more promoters (2913) were differentially methylated between mock and infected samples in comparison to the susceptible cultivar Westar (1775) (Figure 2). There are many studies that also present the plasticity of the occurrence of DNA methylation in response to biotic and abiotic stresses. For example, a genome-wide DNA methylation study in Medicago truncatula under salinity stress shows DNA methylation modification is occurring in different regions of genes with a potential role in salt tolerance responses [36] and there is occurrence of DNA methylation modification in different regions of genes in B. rapa in response to the pathogen Fusarium oxysporum $\mathrm{f}$. sp. conglutinans [37].

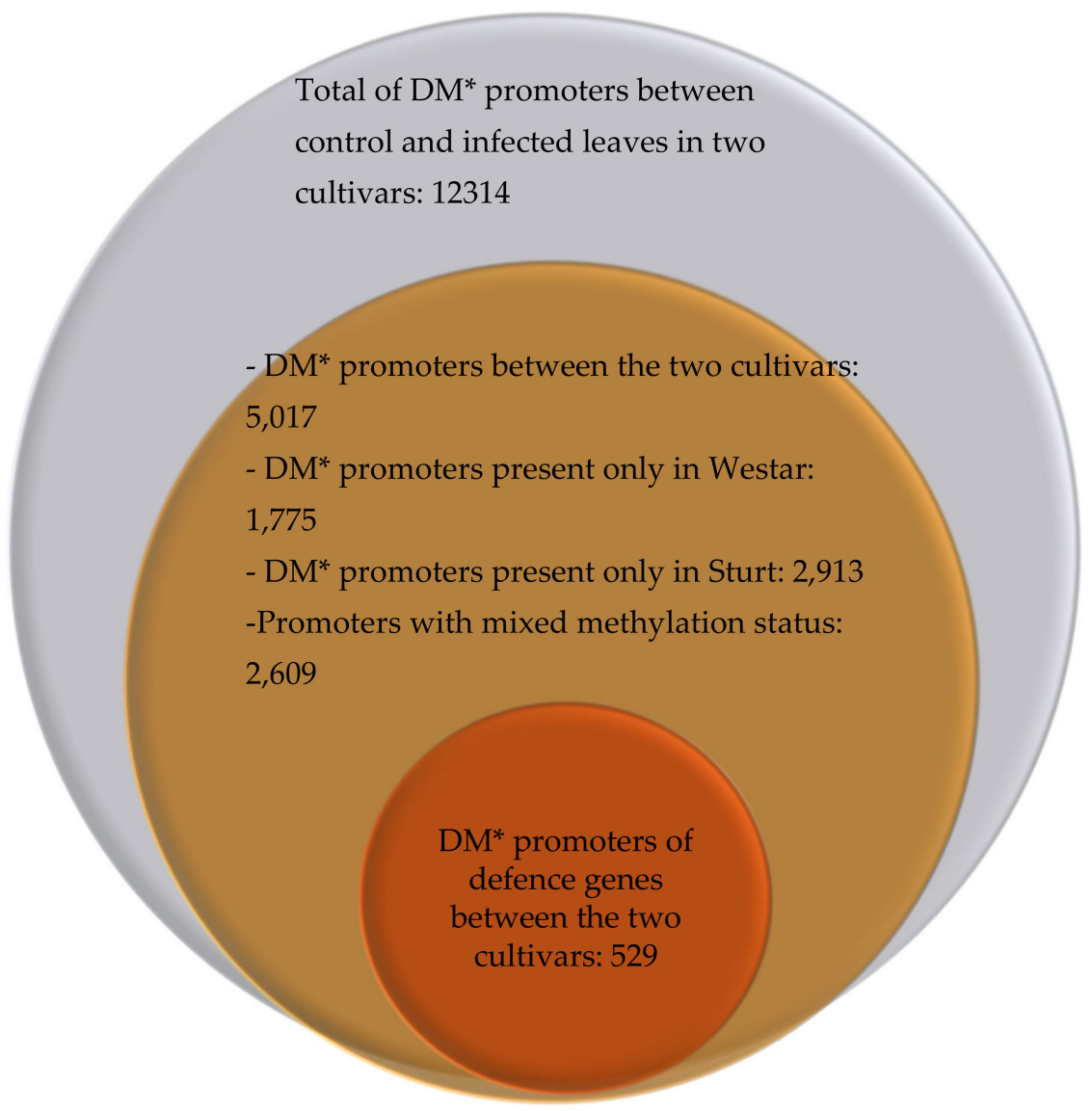

Figure 2. Total number of differentially methylated promoters between leaves from control plants and leaves from infected plants with Leptosphaeria maculans of B. napus cultivars Westar (susceptible) and Sturt (resistant). *Differentially Methylated.

Here, the context percent of the differentially methylated bases between leaves from control plants and leaves from infected plants were also investigated in both cultivars (Table 1). Methylation context is consistent among the leaves and cultivars where the majority of methylation modifications occur in CpG context followed by CHG and CHH in all leaves in both cultivars (Table 1). Similarly, a study of WGBS of B. rapa showed, in a descending order, the methylation modifications happened in CpG, $\mathrm{CHG}$ and $\mathrm{CHH}$ context, in the $2 \mathrm{~kb}$ upstream of the genes [37]. 
Table 1. The context percent of the differentially methylated bases between leaves from control plants and leaves from plants infected with Leptosphaeria maculans of B. napus cultivars Westar (susceptible) and Sturt (resistant) among all differentially methylated promoters and differentially methylated promoters of defence genes.

\begin{tabular}{|c|c|c|c|c|c|c|c|}
\hline & Sturt_Leaf 1 & Sturt_Leaf 2 & Sturt_Leaf 3 & Sturt_Leaf 4 & Westar_Leaf 1 & Westar_Leaf 2 & Westar_Leaf 3 \\
\hline \multicolumn{8}{|c|}{ All differentially methylated promoters } \\
\hline $\mathrm{CpG} \%$ & 93 & 91 & 92 & 92 & 90 & 90 & 90 \\
\hline CHG\% & 5 & 7 & 6 & 6 & 7 & 7 & 7 \\
\hline $\mathrm{CHH} \%$ & 2 & 2 & 2 & 2 & 3 & 3 & 3 \\
\hline \multicolumn{8}{|c|}{ Differentially methylated promoters of defence genes } \\
\hline $\mathrm{CpG} \%$ & 96 & 96 & 96 & 96 & 94 & 94 & 94 \\
\hline CHG\% & 2 & 3 & 3 & 3 & 4 & 5 & 5 \\
\hline $\mathrm{CHH} \%$ & 2 & 1 & 1 & 1 & 2 & 1 & 1 \\
\hline
\end{tabular}

\subsection{Differentially Methylated Promoters of Defence Genes in Westar and Sturt Cultivars Under Pathogen Attack}

Genes located in the downstream of the differentially methylated promoters were searched for genes with gene ontology terms related to defence mechanisms, including the GO term of defence response (GO:0006952), cellular response to stress (GO:0033554) and response to stress (GO:0006950). However, no genes were identified under cellular response to stress (GO:0033554) and response to stress (GO:0006950) GO terms, and only the GO term of defence response (GO:0006952) was identified among these genes. In potato, genes linked to the GO term of defence response (GO:0006952) were confirmed to be responsive to pathogen attack since their expression pattern changed significantly in response to the pathogen Phytophthora infestans [38]. We also investigated if the differentially methylated promoters were located in the upstream region of RGAs (see Section 3.4). The results show, from the total of 12,314 differentially methylated promoters, $529(4.29 \%)$ were located upstream of defence related genes (Figure 2 and Table S1). From these 529 differentially methylated promoters, including 262 promoters that were only differentially methylated in one of the cultivars, 42 promoters were differentially methylated in both cultivars and 225 promoters were differentially methylated between the resistant and susceptible cultivars. Out of 262 promoters, 172 of them were differentially methylated only in Sturt, where 45 were hypo-methylated, 68 hyper-methylated and 59 had mixed methylation status (i.e., in some leaves hypo-methylated and in some leaves hyper-methylated). The remaining 90 promoters were only identified in Westar, including 30 hypo-methylated promoters; 40 hyper-methylated promoters; and 20 promoters with mixed methylation status (Figure 3). In general, DNA methylation modification occurs more across promoters of defence genes in the resistant cultivar Sturt (439 promoters) than promoters in the susceptible cultivar Westar (357 promoters). In line with our previous observation, this suggests that in the resistant cultivar Sturt the occurrence of DNA methylation modification is more dynamic under pathogen attack in comparison to the susceptible cultivar Westar and that these DNA methylation modifications can potentially be responsible for the regulation of resistance-related genes.

Another investigation was performed on corresponding defence genes of the 225 promoters that were differentially methylated between Sturt and Westar. The methylation context of these promoters was similar to the methylation context of other genes promoters, and no significant increases were observed in any of the contexts ( $\mathrm{CpG}, \mathrm{CHH}$ and $\mathrm{CHG}$ ). However, interestingly most of these promoters (55.11\%) were hypo-methylated in Westar and hyper-methylated in Sturt. In general, promoter hypo-methylation results in gene upregulation and has been previously reported among resistance genes. For example, in rice hypo-methylation of the promoter region of Xa21G, a Xanthomonas oryzae pv.oryzae resistance gene resulted in a high level of gene expression and subsequently the resistant phenotype to the pathogen [25]. However, hypo-methylation does not always result in upregulation. $\mathrm{Li}$ et al. (2011) showed that rice hypo-methylation in the promoter of Pib, an NLR, reduced the 
expression level and subsequently compromised the resistant phenotype against the Magnaporthe grisea pathogen [23].

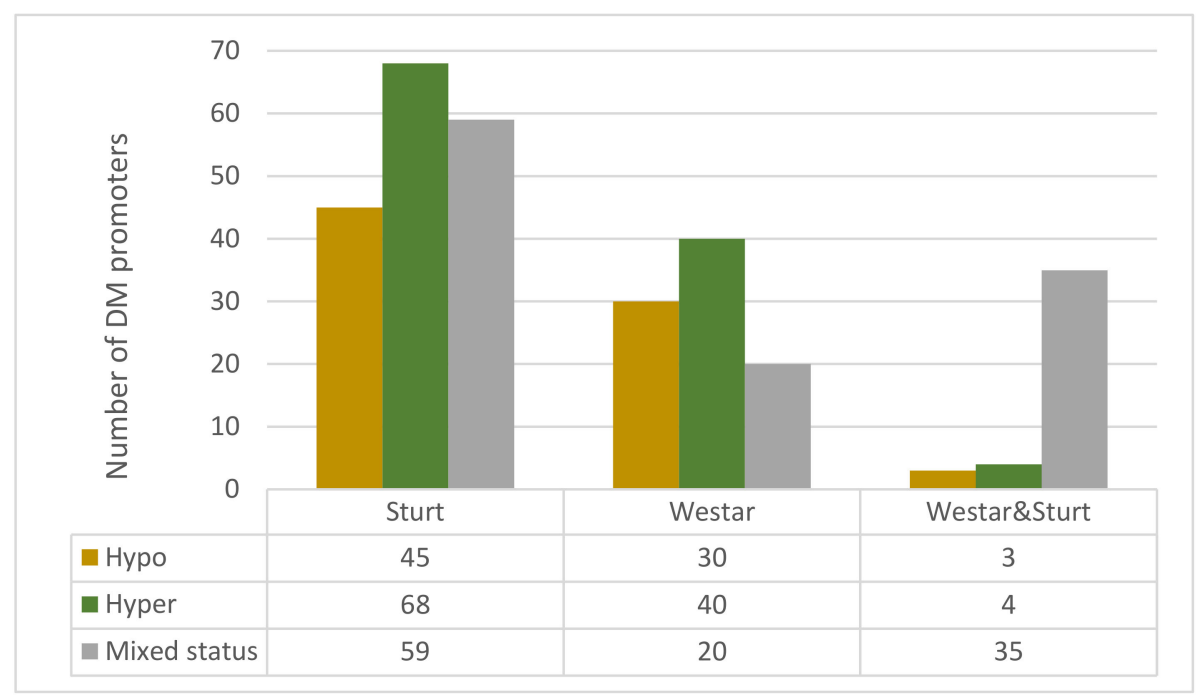

Figure 3. Number of differentially methylated promoters of defence genes between leaves from control plants and leaves from infected plants with Leptosphaeria maculans of B. napus cultivars Westar (susceptible) and Sturt (resistant). Hypo: hypo-methylated, Hyper: hyper-methylated, Mixed status: in some leaves hypo-methylated and in some leaves hyper-methylated, DM: differentially methylated.

It has been suggested that DNA hypo-methylation promotes an increase in somatic recombination in tissues exposed to pathogens [39] and influences the expression of defence genes [10]. Low methylation levels, by increasing the rate of mutation, will increase the evolution of resistance genes under selective pressure in the long term [28]. On the other hand, a high level of methylation will decrease transcription, reducing energy consumption of the cell, which is necessary during pathogenic attack or abiotic stresses [40]. Inappropriate expression of defence genes can be detrimental to plant growth, for example, in Arabidopsis, upregulation of an NLR gene induced by a ddm1(decrease in DNA methylation1) mutant caused a dwarf phenotype [41]. In this regard, it is important to understand the plant's strategy for gene expression regulation of defence genes to better use these strategies toward resistance improvement.

The results show that across the 225 promoters of defence genes, DNA methylation changes occur in all three sequence contexts ( $\mathrm{CpG}, \mathrm{CHG}$ and $\mathrm{CHH})$, while the majority of these changes happened in the $\mathrm{CpG}$ context in both cultivars across all leaves (Table 1). The fact that most of the DNA methylation changes occur in the CpG context suggests that DNA methylation of CpG might have a crucial role in gene regulation and subsequently in the resistance response in B. napus. Similarly, in watermelon leaf samples infected with Cucumber green mottle mosaic virus (CGMMV), the highest level of DNA methylation occurred in the CpG context in $2 \mathrm{kbp}$ upstream, gene bodies, introns and $2 \mathrm{kbp}$ downstream of genes [42]. In rice, two regions within the promoter of the NLR gene Pib were also heavily methylated in CpG context (the methylation level of these regions regulates the expression level of Pib genes and subsequently control the resistance response against Magnaporthe grisea pathogen [23]).

\subsection{Differentially Methylated Promoters of RGAs in Westar and Sturt Cultivars Under Pathogen Attack}

Out of 262 promoters that were only differentially methylated in one of the cultivars, 98 (37.4\%) were RGAs, including 3 TNL, 4 CNL, 3 CN, 2 NBS, 4 NL, 4 TN, 3 TX, 38 RLK, 10 RLP and 27 TM-CC. From 225 promoters that were differentially methylated between the resistant and susceptible cultivars, 87 (38.66\%) were promoters of RGAs, including 4 NBS, 39 RLK, 15 TM-CC, 14 RLP, 8 NL, 3 CNL, 1 TNL, $1 \mathrm{TN}$ and 1 TX genes (Table S1). Out of these 87 RGA promoters, 45 (51.72\%) were hypo-methylated in 
Westar and hyper-methylated in Sturt. The remaining 42 (48.27\%) promoters were hyper-methylated in Westar and hypo-methylated in Sturt (Table S1). RLKs are involved in different biological processes, including defence mechanisms, where they are involved in both recognition and signalling pathways. Among all classes of RGAs, plant genomes carry the largest number of RLKs, such as in species from the Brassicaceae family $[31,35,43]$. The largest number of differentially methylated promoters of RLKs in comparison to other RGA classes can partially be due to the importance of RLKs in the resistance response and activating plant innate immunity and can partially be explained by their abundance in the genome and their multifunctionality. The methylation pattern of both RLK and RLPs also changed under pathogen (CGMMV) attack in watermelon leaves [42]. DNA methylation modification of RLKs has been proven to occur in response to gamma radiation stresses and caused the observed variations in the intensity of the red colour in both skin and interior of the syconiums under stress conditions [44].

There are a number of studies showing that DNA methylation changes in promoter regions of NLRs that play a crucial role in resistance response to pathogens. Yang et al. (2013) reported that DNA methylation variation in a promoter of a CNL in $M$. truncatula resulted in production of resistant (MtREP1) and susceptible (Mtrep1) alleles of this gene, suggesting the potential role of epialleles in the regulation of disease resistance genes [45]. In addition, DNA methylation modification of a promoter of a TNL in Arabidopsis [46] and a promoter of a CNL in rice [24] is also reported to be responsible for resistance response against pathogens. These observations, along with our results, strongly suggest that DNA methylation in promoters of NLR genes confers an important role in regulating defence mechanisms. Here, we also performed a Chi-Square test to examine the association between the occurrence of DNA methylation modification on promoters of RGA and the class of RGA; the outcome showed that associations were statistically significant $(p>0.05)$ for none of the RGA classes, indicating methylation modification is not correlated with RGA classes.

B. napus contains 2252 RGAs, of which 87 (3.86\%) were differentially methylated and distributed across the $B$. napus sub-genomes (A and $\mathrm{C}$ ). The results show the majority of differentially methylated RGAs (51) are located in sub genome $C$, where chromosome $C 08$ contains the largest number of 10 RGAs. Thirty six of the differentially methylated RGAs are located in sub genome A, where chromosome A09 contains the largest number (8 RGAs) (Table S1). This observation can be explained by the fact that the $C$ sub genome of $B$. napus harbours a higher number of resistance genes in comparison to the A sub genome $[31,47]$.

\subsection{Comparative Analysis of Differentially Methylated Promoters of Defence Genes Among Leaves}

Comparative analysis of the 225 promoters of defence genes was performed among the leaf samples to investigate the methylation changes in promoters of defence genes as plants grow. At the time of sampling ( $9 \mathrm{dpi})$, Westar and Sturt cultivars had three and four true leaves, respectively, and all leaves were harvested and their methylation status investigated separately. The first and second leaves in both cultivars refer to the oldest leaves, which means they have the longest time available to defend against the pathogen.

The results show that in both cultivars the majority of promoters of defence genes are hyperand hypo-methylated in the first and second true leaves (Figure 4), while the number of differentially methylated promoters of defence genes substantially decrease in the third and fourth true leaves. Only two promoters were hyper-methylated specifically in the third leaf of Westar, and in Sturt in total only eight promoters were hyper-methylated specifically in the third and fourth leaves (Figure 4). These observations suggest pathogen attack at the cotyledon stage is activating the defence mechanisms in leaves; however, as the plant grows new leaves the defence mechanisms are slowed down. Interestingly, despite the number of responsive genes in both cultivars decreasing, in the youngest leaf in the susceptible cultivar (Westar), the drop was more significant where from 225 only three $(1.33 \%)$ promoters of defence genes were differentially methylated in leaf 3 , while in the resistant cultivar $44(19.55 \%)$ promoters of defence genes were differentially methylated in leaf 4 . Moreover, in Sturt $8.88 \%$ of promoters were continuously hyper- (16 promoters) and hypo- (four promoters) 
methylated in all four leaves; however, only one promoter (0.4\%) stayed hyper-methylated in all three leaves of Westar. These results demonstrate that the defence mechanisms stay activated for a longer time in the resistant cultivar, which potentially prevents the disease from spreading through the plant/stem resulting in less damage at the adult stage.
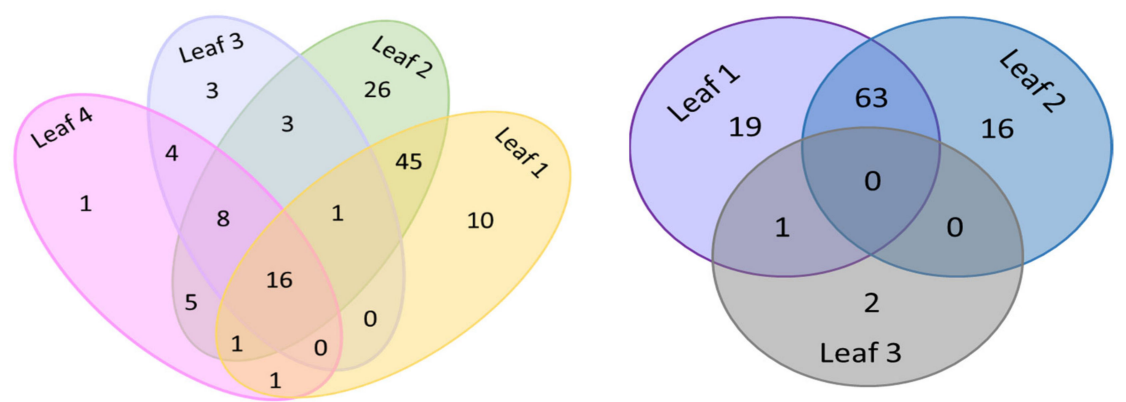

(A)

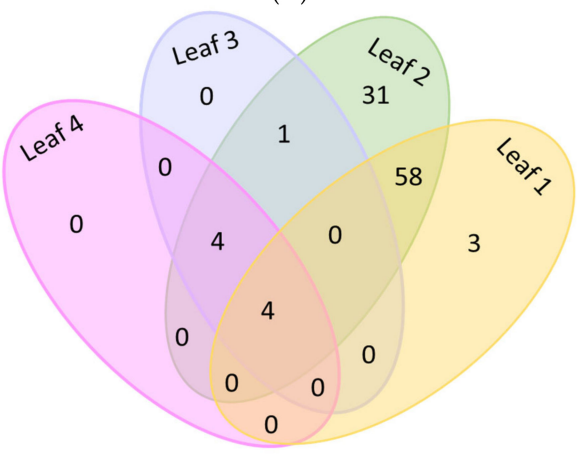

(C)

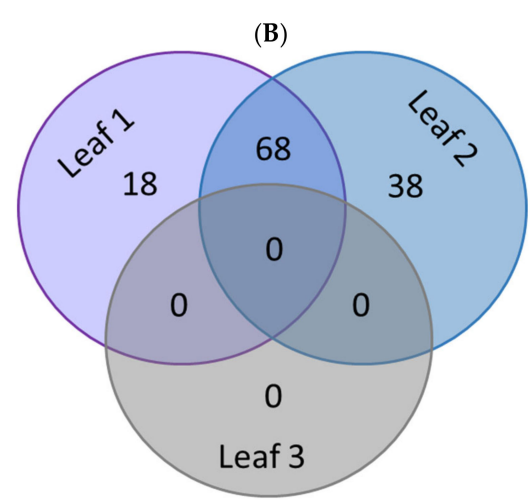

(D)

Figure 4. Venn diagram of the numbers of differentially methylated promoters of defence genes between leaves from control plants and leaves from infected plants with Leptosphaeria maculans of B. napus cultivars Westar (susceptible) and Sturt (resistant). Hyper-methylation in Sturt (A) and Westar (B); Hypo-methylation in Sturt (C) and Westar (D).

\section{Conclusions}

DNA methylation is one of the regulatory mechanisms of defence genes. Here, we show that a large number of promoters of RGAs are differentially methylated in resistant and susceptible cultivars of $B$. napus in response to blackleg disease. While considering the number of differentially methylated promoter of defence genes, DNA methylation modifications were more dynamic in Sturt (resistant) under pathogen pressure, where more promoters of defence genes were differentially methylated in comparison to Westar (susceptible). Additionally, we found most of the promoters of defence genes were hyper-methylated in the resistant cultivar. It has been reported in rice that hyper-methylation of resistance gene promoters is needed for resistance response and upregulation of resistance genes [23]; however, further investigation is required to better understand the B. napus defence strategy against blackleg. We also show that the majority of DNA methylation modification in promoters of both cultivars occurs in the CpG context, suggesting the potential role of CpG DNA methylation in promoter regulation of defence genes. The results also reveal that resistance cultivar defence mechanisms stay activated for longer, as in the youngest leaves of Sturt (resistant cultivar) more promoters of defence genes were differentially methylated compared to Westar (susceptible cultivar). This resistance strategy used by a resistant cultivar can prevent the disease spreading through the plant at the adult stage and result in less damage and yield loss. The outcomes of this study advance our understanding of the role of DNA methylation in plant defence against disease and ultimately help breeders to optimise the breeding programs for enhanced resistance against blackleg disease by including epigenetic modifications. 
Supplementary Materials: The following are available online at http://www.mdpi.com/2073-4395/10/8/1072/s1, Table S1: List of genes located upstream of deferentially methylated promoters between leaves from control plants and leaves from plants infected with Leptosphaeria maculans of Brassica napus cultivars Westar (susceptible) and Sturt (resistant).

Author Contributions: S.T., D.E. and J.B. conceived the study. S.T., C.M. and P.E.B. performed data analysis. S.T and A.A.S.-E. performed laboratory procedures. S.T. wrote the manuscript. J.B. commented on and revised the manuscript. All authors have read and agreed to the published version of the manuscript.

Funding: S.T. is supported by University of Western Australia and Grains Research and Development Corporation (No. 9175960).

Acknowledgments: S.T. acknowledges the supported of University of Western Australia and Grains Research and Development Corporation (No. 9175960). P.E.B. acknowledges support of the Forrest Research Foundation. The authors would like to thank Angela Van De Wouw, from School of BioSciences, the University of Melbourne, Victoria, Australia for providing L. maculans isolates. This work was funded by Australian Research Council Projects FT130100604, DP1601004497, LP140100537, LP160100030.

Conflicts of Interest: The authors declare no conflict of interest.

\section{Abbreviations}

\begin{tabular}{|c|c|}
\hline Avr gene & Avirulence gene \\
\hline AvrLm & Avirulence to Leptosphaeria maculans \\
\hline $\mathrm{CC}$ & Coiled-Coil domain \\
\hline CpG & Cytosine-Guanine \\
\hline $\mathrm{CHG}$ & Cytosine- H(Cytosine/Thymine/Adenine)- Guanine \\
\hline $\mathrm{CHH}$ & Cytosine- H(Cytosine/Thymine/Adenine)- H(Cytosine/Thymine/Adenine) \\
\hline CNL & CC-NBS-LRR \\
\hline DMR & Differentially methylated region \\
\hline DNA & Deoxyribonucleic acid \\
\hline ETI & Effector-Triggered Immunity \\
\hline GO & Gene Ontology \\
\hline LRR & Leucine Rich Repeat \\
\hline RLK & Receptor-Like Kinase \\
\hline RLP & Receptor-Like Proteins \\
\hline NBS & Nucleotide Binding Site \\
\hline NLR & Nucleotide Binding Site- Leucine Rich Repeat \\
\hline PAMP & Pathogen-associated molecular pattern \\
\hline PTI & PAMP-triggered immunity \\
\hline R gene & Resistance gene \\
\hline RGA & Resistance Gene Analog \\
\hline RLP & Receptor-Like Proteins \\
\hline $\mathrm{TE}$ & Transposable Elements \\
\hline TIR & Toll/Interleukin-1 Receptor domain \\
\hline TNL & TIR-NBS-LRR \\
\hline WGBS & Whole genome bisulfite sequencing \\
\hline
\end{tabular}

\section{References}

1. Kole, C. Genome Mapping and Molecular Breeding in Plants; Springer: Berlin, Germany, 2007.

2. Zhang, X.; Fernando, W.D. Insights into fighting against blackleg disease of Brassica napus in Canada. Crop. Pasture Sci. 2018, 69, 40-47. [CrossRef]

3. Howlett, B.J.; Idnurm, A.; Pedras, M.S.C. Leptosphaeria maculans, the causal agent of blackleg disease of Brassicas. Fungal Genet. Biol. 2001, 33, 1-14. [CrossRef]

4. Sprague, S.J.; Marcroft, S.J.; Hayden, H.L.; Howlett, B.J. Major gene resistance to blackleg in Brassica napus overcome within three years of commercial production in southeastern Australia. Plant. Dis. 2006, 90, 190-198. [CrossRef] [PubMed]

5. Sekhwal, M.K.; Li, P.; Lam, I.; Wang, X.; Cloutier, S.; You, F.M. Disease resistance gene analogs (RGAs) in plants. Int. J. Mol. Sci. 2015, 16, 19248-19290. [CrossRef] [PubMed] 
6. Jones, J.D.G.; Dangl, J.L. The plant immune system. Nature 2006, 444, 323-329. [CrossRef]

7. Tsuda, K.; Katagiri, F. Comparing signaling mechanisms engaged in pattern-triggered and effector-triggered immunity. Curr. Opin. Plant. Biol. 2010, 13, 459-465. [CrossRef] [PubMed]

8. Flor, H.H. Current status of the gene-for-gene concept. Annu. Rev. Phytopathol. 1971, 9, 275-296. [CrossRef]

9. Yang, X.; Deng, F.; Ramonell, K.M. Receptor-like kinases and receptor-like proteins: Keys to pathogen recognition and defense signaling in plant innate immunity. Front. Biol. 2012, 7, 155-166. [CrossRef]

10. Zhu, Q.-H.; Shan, W.-X.; Ayliffe, M.A.; Wang, M.-B. Epigenetic mechanisms: An emerging player in plant-microbe interactions. Mol. Plant.-Microbe Interact. 2016, 29, 187-196. [CrossRef]

11. Dowen, R.H.; Pelizzola, M.; Schmitz, R.J.; Lister, R.; Dowen, J.M.; Nery, J.R.; Dixon, J.E.; Ecker, J.R. Widespread dynamic DNA methylation in response to biotic stress. PNAS USA 2012, 109, E2183-E2191. [CrossRef]

12. Finnegan, E.J.; Peacock, W.J.; Dennis, E.S. DNA methylation, a key regulator of plant development and other processes. Curr. Opin. Genet. Dev. 2000, 10, 217-223. [CrossRef]

13. Chan, S.W.L.; Henderson, I.R.; Jacobsen, S.E. Gardening the genome: DNA methylation in Arabidopsis thaliana. Nat. Rev. Genet. 2005, 6, 351-360. [CrossRef] [PubMed]

14. Colot, V.; Rossignol, J.L. Eukaryotic DNA methylation as an evolutionary device. BioEssays 1999, $21,402-411$. [CrossRef]

15. Henderson, I.R.; Jacobsen, S.E. Epigenetic inheritance in plants. Nature 2007, 447, 418-424. [CrossRef] [PubMed]

16. Elhamamsy, A.R. DNA methylation dynamics in plants and mammals: Overview of regulation and dysregulation. Cell Biochem. Funct. 2016, 34, 289-298. [CrossRef] [PubMed]

17. Tirnaz, S.; Batley, J. DNA methylation: Toward crop disease resistance improvement. Trends Plant. Sci. 2019, 24, 1137-1150. [CrossRef] [PubMed]

18. Tirnaz, S.; Batley, J. Epigenetics: Potentials and challenges in crop breeding. Mol. Plant. 2019, 12, 1309-1311. [CrossRef]

19. Wang, J.; Marowsky, N.C.; Fan, C. Divergence of gene body DNA methylation and evolution of plant duplicate genes. PLoS ONE 2014, 9, e110357. [CrossRef]

20. Do Kim, K.; El Baidouri, M.; Abernathy, B.; Iwata-Otsubo, A.; Chavarro, C.; Gonzales, M.; Libault, M.; Grimwood, J.; Jackson, S.A. A comparative epigenomic analysis of polyploidy-derived genes in soybean and common bean. Plant. Physiol. 2015, 168, 1433-1447. [CrossRef]

21. Wang, X.; Zhang, Z.; Fu, T.; Hu, L.; Xu, C.; Gong, L.; Wendel, J.F.; Liu, B. Gene-body CG methylation and divergent expression of duplicate genes in rice. Sci. Rep. 2017, 7, 2675. [CrossRef]

22. Li, X.; Zhu, J.; Hu, F.; Ge, S.; Ye, M.; Xiang, H.; Zhang, G.; Zheng, X.; Zhang, H.; Zhang, S. Single-base resolution maps of cultivated and wild rice methylomes and regulatory roles of DNA methylation in plant gene expression. BMC Genom. 2012, 13, 300. [CrossRef] [PubMed]

23. Li, Y.; Xia, Q.; Kou, H.; Wang, D.; Lin, X.; Wu, Y.; Xu, C.; Xing, S.; Liu, B. Induced Pib expression and resistance to Magnaporthe grisea are compromised by cytosine demethylation at critical promoter regions in rice. J. Integr. Plant. Biol. 2011, 53, 814-823. [CrossRef] [PubMed]

24. Deng, Y.; Zhai, K.; Xie, Z.; Yang, D.; Zhu, X.; Liu, J.; Wang, X.; Qin, P.; Yang, Y.; Zhang, G. Epigenetic regulation of antagonistic receptors confers rice blast resistance with yield balance. Science 2017, 355, 962-965. [CrossRef] [PubMed]

25. Akimoto, K.; Katakami, H.; Kim, H.-J.; Ogawa, E.; Sano, C.M.; Wada, Y.; Sano, H. Epigenetic inheritance in rice plants. Ann. Bot. 2007, 100, 205-217. [CrossRef]

26. Karan, R.; DeLeon, T.; Biradar, H.; Subudhi, P.K. Salt stress induced variation in DNA methylation pattern and its influence on gene expression in contrasting rice genotypes. PLoS ONE 2012, 7, e40203. [CrossRef]

27. Liu, G.; Xia, Y.; Liu, T.; Dai, S.; Hou, X. The DNA methylome and association of differentially methylated regions with differential gene expression during heat stress in Brassica rapa. Int. J. Mol. Sci. 2018, 19, 1414. [CrossRef]

28. Boyko, A.; Kovalchuk, I. Genetic and epigenetic effects of plant-pathogen interactions: An evolutionary perspective. Mol. Plant. 2011, 4, 1014-1023. [CrossRef]

29. Purwantara, A.; Salisbury, P.A.; Burton, W.A.; Howlett, B.J. Reaction of Brassica juncea (Indian Mustard) Lines to Australian Isolates of Leptosphaeria maculans under Glasshouse and Field Conditions. Eur. J. Plant. Pathol. 1998, 104, 895-902. [CrossRef] 
30. Bao, W.; Kojima, K.K.; Kohany, O. Repbase update, a database of repetitive elements in eukaryotic genomes. Mob. DNA 2015, 6, 11. [CrossRef]

31. Hurgobin, B.; Golicz, A.A.; Bayer, P.E.; Chan, C.-K.K.; Tirnaz, S.; Dolatabadian, A.; Schiessl, S.V.; Samans, B.; Montenegro, J.D.; Parkin, I.A.P.; et al. Homoeologous exchange is a major cause of gene presence/absence variation in the amphidiploid Brassica napus. Plant. Biotechnol. J. 2018, 16, 1265-1274. [CrossRef]

32. Bayer, P.E.; Hurgobin, B.; Golicz, A.A.; Chan, C.K.K.; Yuan, Y.; Lee, H.; Renton, M.; Meng, J.; Li, R.; Long, Y. Assembly and comparison of two closely related Brassica napus genomes. Plant. Biotechnol. J. 2017, 15, 1602-1610. [CrossRef]

33. Krueger, F.; Andrews, S.R. Bismark: A flexible aligner and methylation caller for Bisulfite-Seq applications. Bioinformatics 2011, 27, 1571-1572. [CrossRef] [PubMed]

34. Li, P.; Quan, X.; Jia, G.; Xiao, J.; Cloutier, S.; You, F.M. RGAugury: A pipeline for genome-wide prediction of resistance gene analogs (RGAs) in plants. BMC Genom. 2016, 17, 852. [CrossRef] [PubMed]

35. Tirnaz, S.; Bayer, P.; Inturrisi, F.; Neik, T.; Yang, H.; Dolatabadian, A.; Zhang, F.; Severn-Ellis, A.; Patel, D.; Pradhan, A.; et al. Resistance gene analogs in the Brassicaceae: Identification, characterization, distribution and evolution. Plant. Physiol. 2020, in press.

36. Yaish, M.W.; Al-Lawati, A.; Al-Harrasi, I.; Patankar, H.V. Genome-wide DNA Methylation analysis in response to salinity in the model plant caliph medic (Medicago truncatula). BMC Genom. 2018, 19, 78. [CrossRef]

37. Takahashi, S.; Fukushima, N.; Osabe, K.; Itabashi, E.; Shimizu, M.; Miyaji, N.; Takasaki-Yasuda, T.; Suzuki, Y.; Seki, M.; Fujimoto, R. Identification of DNA methylated regions by using methylated DNA immunoprecipitation sequencing in Brassica rapa. Crop. Pasture Sci. 2018, 69, 107-120. [CrossRef]

38. Gyetvai, G.; Sønderkær, M.; Göbel, U.; Basekow, R.; Ballvora, A.; Imhoff, M.; Kersten, B.; Nielsen, K.-L.; Gebhardt, C. The transcriptome of compatible and incompatible interactions of potato (Solanum tuberosum) with Phytophthora infestans revealed by DeepSAGE analysis. PLoS ONE 2012, 7, e31526. [CrossRef]

39. Alvarez, M.E.; Nota, F.; Cambiagno, D.A. Epigenetic control of plant immunity. Mol. Plant. Pathol. 2010, 11, 563-576. [CrossRef]

40. Yaish, M.W. DNA methylation-associated epigenetic changes in stress tolerance of plants. In Molecular Stress Physiology of Plants; Rout, G.R., Das, A.B., Eds.; Springer: New Delhi, India, 2013; pp. 427-440.

41. Stokes, T.L.; Kunkel, B.N.; Richards, E.J. Epigenetic variation in Arabidopsis disease resistance. Genes Dev. 2002, 16, 171-182. [CrossRef]

42. Sun, Y.; Fan, M.; He, Y. DNA methylation analysis of the Citrullus lanatus response to Cucumber Green Mottle Mosaic Virus infection by whole-genome bisulfite sequencing. Genes 2019, 10, 344. [CrossRef]

43. Bayer, P.E.; Golicz, A.A.; Tirnaz, S.; Chan, C.K.K.; Edwards, D.; Batley, J. Variation in abundance of predicted resistance genes in the Brassica oleracea pangenome. Plant. Biotechnol. J. 2019, 17, 789-800. [CrossRef] [PubMed]

44. Rodrigues, M.G.F.; Soares, M.R.; Gimenez, D.F.J.; Fonseca, L.F.S.; Torrieri, E.; Ramos, E.S.; Giuliatti, S. Detection of differentially methylated regions of irradiated fig tree selections. Sci. Agric. 2017, 74, 285-293. [CrossRef]

45. Yang, S.; Tang, F.; Caixeta, E.T.; Zhu, H. Epigenetic regulation of a powdery mildew resistance gene in Medicago truncatula. Mol. Plant. 2013, 6, 2000-2003. [CrossRef]

46. Yu, A.; Lepère, G.; Jay, F.; Wang, J.; Bapaume, L.; Wang, Y.; Abraham, A.-L.; Penterman, J.; Fischer, R.L.; Voinnet, $\mathrm{O}$. Dynamics and biological relevance of DNA demethylation in Arabidopsis antibacterial defense. PNAS USA 2013, 110, 2389-2394. [CrossRef] [PubMed]

47. Alamery, S.; Tirnaz, S.; Bayer, P.; Tollenaere, R.; Chaloub, B.; Edwards, D.; Batley, J. Genome-wide identification and comparative analysis of NBS-LRR resistance genes in Brassica napus. Crop. Pasture Sci. 2018, 69, 72-93. [CrossRef]

(C) 2020 by the authors. Licensee MDPI, Basel, Switzerland. This article is an open access article distributed under the terms and conditions of the Creative Commons Attribution (CC BY) license (http://creativecommons.org/licenses/by/4.0/). 